\title{
Research of the effect of aberrations on image quality in optical systems
}

\author{
A.V. Kozhevnikov ${ }^{1}$

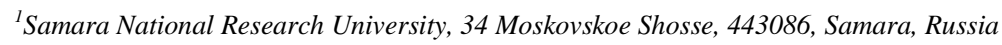

\begin{abstract}
This work presents a study of the influence and compensation of aberrations in optical imaging systems by superimposing surfaces described by Zernike polynomials. The aim is to investigate how to compensate for aberrations and their effectiveness when dealing with aberrations of different types. The study is done in the Zemax packet by modeling the optical imaging system and passing test images through it.
\end{abstract}

Keywords: wave aberrations; Zernike polynomials; imaging optical system

\section{Introduction}

The aberration of the optical system is the deformation of images that occur at the output of the optical system. The name comes from the lat. Aberratio - evasion, removal. Deformation consist in the fact that the optical images do not completely correspond to the object. This is manifested in the blurriness of the image and is called monochromatic geometric aberration or color image - chromatic aberration of the optical system. Most often, both types of aberration appear together.

In the paraxial region, the optical system works almost perfectly, the dot is represented by a point, and the straight line is a straight line, etc. However, as the point moves away from the optical axis, the rays from it intersect in the image plane not at one point. Thus, a circle of dispersion arises, i.e. there are aberrations. The dimension of the aberration can be determined by calculating the geometric and optical formulas through a comparison of the coordinates of the rays, and also approximately using the formulas of the theory of aberrations [1].

There is a description of the phenomenon of aberration in both the ray theory (deviation from identity is described through geometric aberrations and ray scattering patterns) and in the representations of wave optics (the deformation of a spherical light wave along the path through an optical system is estimated). Usually, ray theory is used to characterize optical systems with large aberrations, otherwise the principles of wave optics are applied.

As a rule, analysis of wave aberrations is performed on the basis of Zernike polynomials [1-4]. In [5-8], it was proposed to use a multi diffractive optical elements for an optical wavefront decomposition on the basis of Zernike polynomials. Moreover, the use of optical elements that are consistent not only with Zernike functions, but also their superpositions, makes it possible to perform optical measurements ensuring the restoration of the shape of the wave front [9-11].

In this work, we investigate the effect of aberrations described by Zernike polynomials on the imaging properties of an optical system using the Zemax packet [12]. The aim of the work is to investigate methods of aberration compensation [13-15] and their effectiveness in dealing with aberrations of different types.

\section{Modeling of wave aberrations by varying the coefficients of a polynomial surface}

The first stage will be modeled several surfaces described by Zernike polynomials. For this part, an optical system consisting of two refractive lenses is used, one of which is the surface under investigation. Light with wavelengths of 450, 550 and $650 \mathrm{~nm}$ is transmitted through such a system. As an estimation of aberration distortions, an image of the Latin letter "F", passed through the described optical system, is considered. The most interesting are the coefficients - "Zernike standard coefficients".

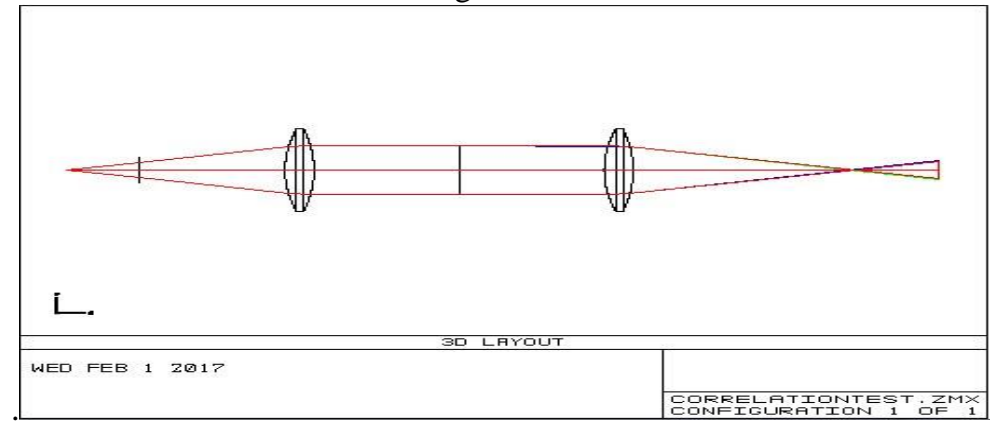

Fig.1. Two-dimensional scheme of the optical system

There are even and odd Zernike polynomials. Even polynomials are defined as: $Z(\rho, \varphi)=R_{n}{ }^{m}(\rho) \cos (m \varphi)$, and odd as: $Z_{n}{ }^{-m}(\rho, \varphi)=R_{n}{ }^{m}(\rho) \sin (m \varphi)$. Where $\mathrm{m}$ and $\mathrm{n}$ are nonnegative integers, such that $\mathrm{n}>\mathrm{m}, \varphi$ - azimuth angle, and $\rho$ - radial distance $0<=\rho<=1$. Zernike polynomials are limited in the range from -1 to +1 . Radial polynomials are defined as: $R_{n}^{m}(\rho)=$ $\sum_{k=0}^{(n-m) / 2} \frac{(-1)^{k}(n-k) !}{k !\left(\frac{n+m}{2}-k\right) !\left(\frac{n-m}{2}-k\right) !} \rho^{n-2 k}$ 
Computer Optics and Nanophotonics / A.V. Kozhevnikov

Table.1. The result of simulation of wave aberrations by the Zernike row decomposition.

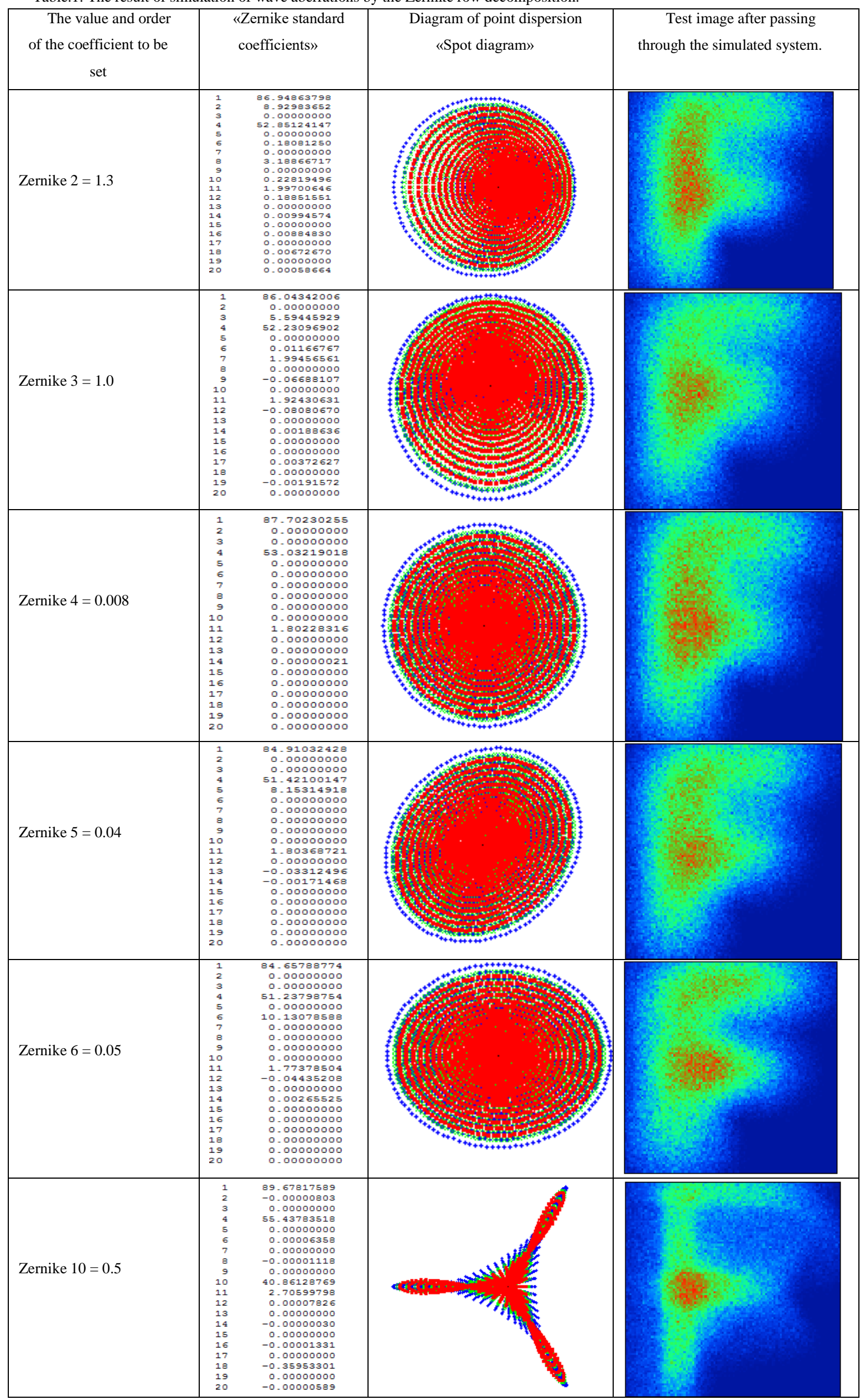


The orthogonality of the Zernike polynomials gives them great advantages when analyzing aberrations in comparison with the exponentiation basis. The main advantages are:

1) The absolute values of the coefficients of the decomposition in the Zernike polynomials decrease with increasing degree of polynomials, that is, the Zernike row, as a rule, always converges, which cannot be said about the exponentiation rows;

2) Each coefficient of the row gives the contribution of aberrations of a given type and order to the total wave aberration from the position of mutual balance of all types of aberrations. This means that the individual types of aberrations represented by the Zernike polynomial decomposition affect image quality quite independently of each other.

Table 2. The result of compensating wave aberrations.

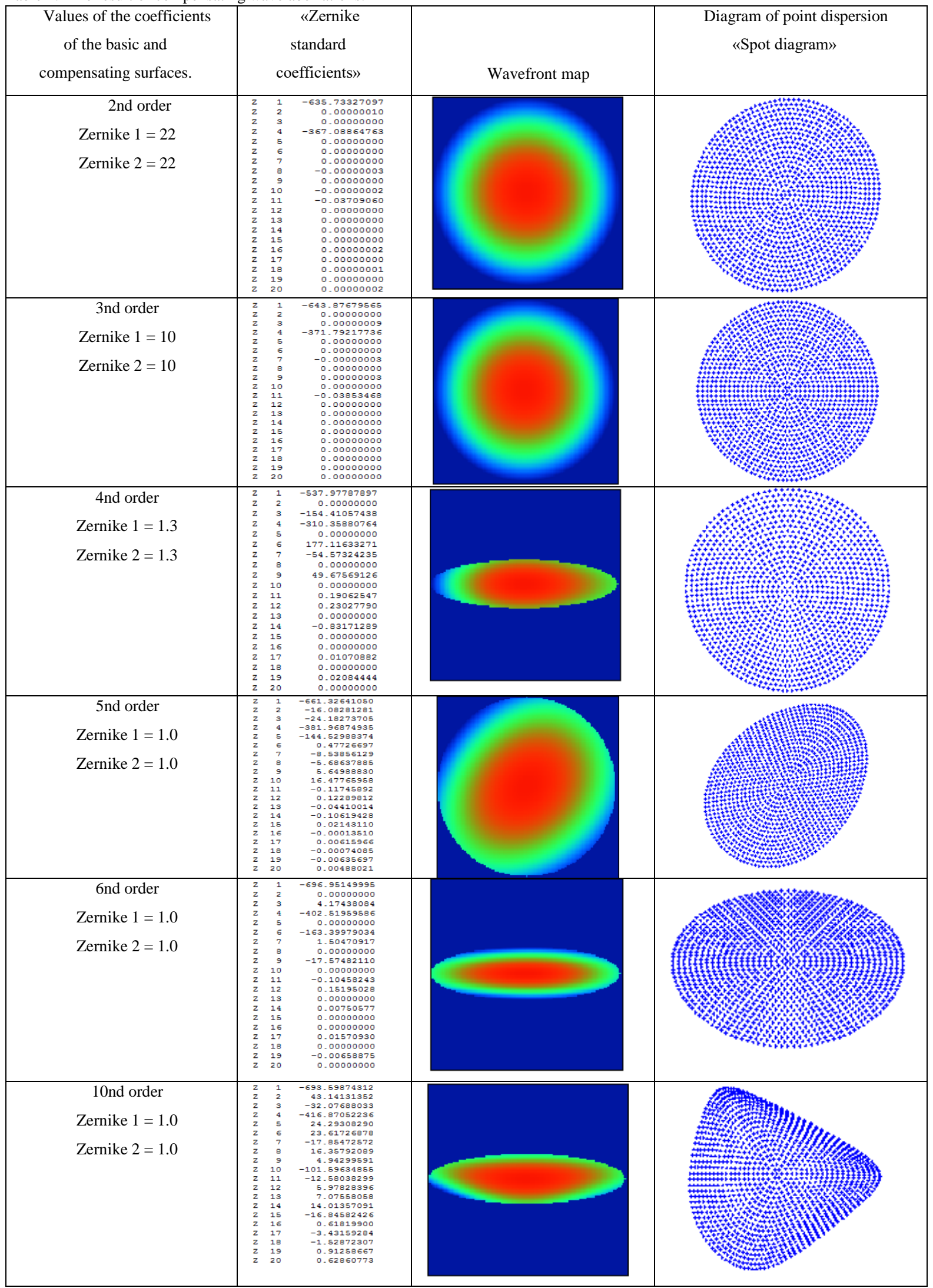


In each case, the coefficients of different orders are taken, from 1st to 10th, they are assigned the maximum values at which the test image preserves the original contour and sharpness. The parameter "Zernike max term" is set to 16, it is responsible for the number of polynomial surface parameters.

As can be seen from Table 1, the variation of the coefficients of the polynomial surface leads to deformations in the point dispersion diagram of and on the test image passed through the test system.

\section{Investigation of compensation of aberrations in the optical system}

In this section, we study the compensation of aberrations in a test optical imaging system by superimposing surfaces described by Zernike polynomials. The aim is to search for the maximum efficiency of compensating wave aberrations by adding a second polynomial surface. The effect is achieved due to the complex conjugacy of polynomials.

The optical system used consists of two mirrors arranged at a certain angle to each other and an auxiliary paraxial surface used to parallelize the incoming light beam. In both mirrors there are polynomially described surfaces.

As can be seen from Table 2, the addition of the second Zernike surface allows us to compensate for the simulated aberrations in the optical system.

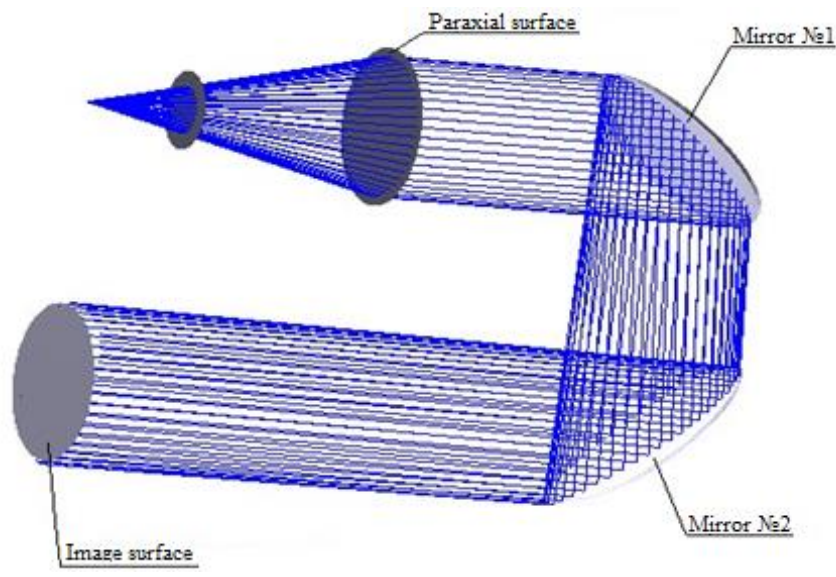

Fig.2. Optical system of two mirrors.

\section{Conclusion}

The decomposition of the wave aberrations in the Zernike row was conducted in this work. Wave aberrations of various types were modeled and their effect on image quality in optical systems was examined. An optical system of two mirrors with two polynomial surfaces is simulated. The first surface was used to model the aberrations themselves, the second was used to compensate them. The principle used is based on the complex conjugacy of polynomials.

The possibility of compensation of wave aberrations by superposition of surfaces described by Zernike polynomials is investigated. The maximum values of aberrations that can be compensated for by relatively effective compensation due to the use of polynomial surfaces were obtained.

The study was carried out in a Zemax packet by modeling test optical imaging systems, and passing test images through them.

\section{Acknowledgments}

The work was supported by the Ministry of Education and Science of the Russian Federation.

\section{References}

[1] Volf E, Born M. Fundamentals of Optics. Moscow: "Nauka" Publisher, 1973. (in Russian)

[2] American National Standards Institute, Inc. American National Standards for Ophthalmics - Methods for Reporting Optical Aberrations of Eyes. ANSI Z80.28, 2004.

[3] Bezdyidyko SN. Optimization of optical systems using orthogonal polynomials. Optics and spectroscopy 1980; 48: 222-224. (in Russian)

[4] Bezdyidyko SN. Methodological aspects of application of Zernike polynomials in computational optics. Materials of the International Conference dedicated to the 90th anniversary of the birth of the Nobel Prize winner Academician A.M. Prokhorov. The fundamental foundations of engineering 2006; 4. (in Russian)

[5] Khonina SN, Kotlyar VV, Soifer VA, Wang Y, Zhao D. Decomposition of a coherent light field using a phase Zernike filter. Proceedings of SPIE 1998; 3573: $550-553$.

[6] Ha Y, Zhao D, Wang Y, Kotlyar VV, Khonina SN, Soifer VA. Diffractive optical element for Zernike decomposition. Proceedings of SPIE 1998; 3557: 191197.

[7] Khonina SN, Kotlyar VV, Wang Ya. Diffractive optical element matched with Zernike basis. Pattern Recognition and Image Analysis 2001; 11(2): 442-445.

[8] Porfirev AP, Khonina SN. Experimental investigation of multi-order diffractive optical elements matched with two types of Zernike functions. Proceedings of SPIE2016; $9807: 9$ p.

[9] Kotlyar VV, Khonina SN, Soifer VA, Wang Y, Zhao D. Coherent field phase retrieval using a phase Zernike filter. Computer Optics, 1997 ; 17 : 43-48. 
[10] Khonina SN, Kotlyar VV, Kirsh DV. Zernike phase spatial filter for measuring the aberrations of the optical structures of the eye. Journal of Biomedical Photonics \& Engineering 2015; 1(2): 146-153.

[11] Khorin PA, Khorina SN, Karsakov AV, Branchevsky SL. Analysis of human eye cornea aberrations. Computer Optics 2016; 40(6): 810-817. (in Russian) DOI: 10.18287/0134-2452-2016-40-6-810-817.

[12] Zemax ${ }^{\circledR}$ User's Guide. Zemax Development Corporation 2005.

[13] Tokovinin A, Heathcote S. DONUT: measuring optical aberrations from a single extrafocal image. Publications of the Astronomical Society of the Pacific 2006; 118(846): 1165-1175.

[14] Booth MJ. Wavefront sensorless adaptive optics for large aberrations. Optics Letters 2007; 32 (1): 5-7.

[15] Klebanov IM, Karsakov AV, Khonina SN, Davydov AN, Polyakov KA. Wave front aberration compensation of space telescopes with telescope temperature field adjustment. Computer Optics 2017; 41(1): 30-36. (in Russian) DOI: 10.18287/0134-2452-2017-41-1-30-36. 